\title{
Feasibility of local anaesthesia for varicocele correction in one-day-surgery setting. A single center experience
}

\author{
Giovannni Saredi ${ }^{1}$, Fabrizio I. Scroppo ${ }^{1}$, Paolo Capogrosso ${ }^{1}$, Giacomo Maria Pirola ${ }^{2}$, Lorenzo Capone ${ }^{2}$, \\ Andrea Pacchetti ${ }^{3}$, Giuseppe Gianesini ${ }^{1}$, Paolo Maggio ${ }^{1}$, Giulio Carcano ${ }^{4,5}$, Federico Dehò ${ }^{1,4}$ \\ ${ }^{1}$ Department of Urology and Andrology; Circolo and Fondazione Macchi Hospital,Varese Italy; \\ ${ }^{2}$ Department of Urology, USL Toscana Sud Est, San Donato Hospital, Arezzo, Italy; \\ ${ }^{3}$ Department of Urology, San Martino Hospital, University of Genova, Italy; \\ ${ }^{4}$ University of Insubria, Varese, Italy; \\ ${ }^{5}$ Department of Surgery; Circolo and Fondazione Macchi Hospital,Varese Italy.
}

\begin{abstract}
Summary Objective: In this study, we compared postoperative outcomes of patients submitted to varicocele correction under general or local anesthesia at a single center.

Methods: All patients underwent varicocele surgical treatment with the Colpi-modified Marmar subinguinal technique.

They were managed with either general (Group A) or local with ileo-inguinal and ileo-hypogastric nerves block (Group B) anesthesia. The two groups were compared in terms of timing of discharge and post-operative pain as assessed with the numeric rating scale (NRS) at both rest and movement (NRSm).

Results: Overall, 63 patients were included with a mean (SD) age of 25 years \pm 5 yrs. The NRS mean score was significantly lower for Group B during the first 4 days after surgery at both rest and movement (all $p<0.05)$. Patients receiving local anesthesia showed a faster time to first urination (210 vs. $240 \mathrm{~min}$; $p=0.02$ ), although the time to discharge was comparable between the two groups (250 vs. $250 \mathrm{~min}$ ).

Conclusions: These results suggest that local anetshesia for varicocele surgical treatment is feasible and provide better pain control and faster recovery after surgery.
\end{abstract}

KEY WORDS: Varicocele; Local anesthesia; Infertility; Recovery.

Submitted 2 July 2020; Accepted 15 October 2020

\section{INTRODUCTION}

Varicocele is defined as dilated and tortuous veins within the pampiniform plexus of the testis $(1,2)$. It is one of the main cause of male infertility and is commonly associated with semen impairment. The prevalence of varicocele is approximately $15-20 \%$ in the general population, $19 \%-41 \%$ in men with primary infertility, and $45 \%-81 \%$ in men with secondary infertility (3).

The pathophysiology of varicocele-associated infertility involves different factors including blood stasis, accumulation of reactive oxygen species at the level of the testis, increased scrotal temperature and reduction of intratesticular testosterone levels $(4,5)$.

Surgical treatment of varicocele includes percutaneous embolization or surgical correction with different techniques including open retroperitoneal high ligation, laparoscopic ligation or subinguinal microsurgical technique (6). Large-scale comparative studies evaluating the outcomes of various varicocele treatment options are currently lacking, and confounding factors such as the experience of the operators, the age of the patients and the severity of varicocele have a significant impact on skewing the post-treatment results $(7,8)$. Among all treatment options, the subinguinal approach combined with anterograde intraoperative sclerosis of venous vessels (Marmar technique modified by Colpi) represents a valid surgical treatment of varicocele (9): this approach exploits the advantages of both the Marmar (10) and the Tauber (11) technique without the need for microscope or fluoroscope, making the operation more simple and less expensive.

Surgical correction of varicocele is usually performed as a one-day surgery due to the low risk of peri-operative morbidity. In this context, the ability to resume normal activities soon after surgery is an important indicator of a successful perioperative experience and may be largely associated with the type of performed anesthesia and with peri-operative pain management (12).

To the best of our knowledge, studies investigating the best type of anesthesia to be performed in patients undergoing varicocele correction are currently lacking. The purpose of this prospective observational study was to evaluate the difference in the timing of discharge and pain control among patients undergoing surgical correction of varicocele with either local or general anesthesia at a single academic center.

\section{Material AND METHOdS}

This is a prospective, observational investigation trial. The study protocol was approved by the Local Ethics committee of the Institution "Ospedale di Circolo e Fondazione Macchi”, Varese, Italy. Afterward, the study protocol was registered on Clinical Trials.gov (ID Number NCT02401087). Inclusion criteria were: male subjects over the age of 18; with ASA I-II scores; with a clinically significant varicocele scheduled for surgical correction. Patients with cognitive impairment or mental retardation; habitual use of opioid analgesics; alterations in the normal values of coagulation or coagulopathies; use of non-steroidal anti-inflammatory drugs in the 5 days pre-

\footnotetext{
No conflict of interest declared.
} 
ceding the intervention; severe liver or kidney failure; lack of informed consent, were excluded.

After obtaining the Informed Consent, 63 patients with fulfilling the inclusion criteria were recruited in a period between June 2017 and July 2018.

Patients were divided into two groups: patients included in "Group A" underwent general anesthesia (32 patients) and patients included in "Group B" underwent local blockage of the ileoinguinal-ileohypogastric nerve (31 patients). The type of anesthesiological protocol was based on the preference of the anesthesiologist.

All patients received light sedation with Midazolam 0.03 $\mathrm{mg} / \mathrm{kg}$ i.v. before entering the operating room. Moreover, they received a fluid load of $10 \mathrm{ml} / \mathrm{Kg} / \mathrm{h}$ during the surgical procedure and another $5-8 \mathrm{ml} / \mathrm{Kg}$ in the postoperative period.

\section{Anesthesiological protocol}

Group A: At the discretion of the anesthesiologist in the operating room, the maintenance of general anesthesia was either with the use of a halogenated agent (Sevoflurane) or in TIVA (Propofol + Remifentanil). For induction, Fentanyl was used as opioid (for general anesthesia conducted with Sevoflurane) and Fentanyl or Remifentanil for anesthesia conducted in TIVA. Ventilation was achieved with laryngeal mask. All patients received Paracetamol $1 \mathrm{~g}$ i.v. as intraoperative analgesia; if the patient complained about pain upon awakening, Ketorolac $30 \mathrm{mg}$ i.v. was administered. Ondansetron $4 \mathrm{mg}$ i.v. was given as an antiemetic before waking up. In addition to the amount of opioid used, the need for additional antiemetics was also reported.

Group B: After identification by ultrasound of the ileoinguinal and ileo-hypogastric nerves, by means of a plane technique, the aforementioned nerves were blocked with 0.5\% Chirocaine $20 \mathrm{ml}$. Afterward, each patient recieved a subcutaneous skin infiltration with 2\% Carbocaine 10 $\mathrm{ml}$ and an endovenous administration with Paracetamol 1 $\mathrm{g}$ and Ketorolac $30 \mathrm{mg}$ as rescue therapy.

Patients of both groups were discharged at home with the following analgesic therapy: Tramadol $37.5 \mathrm{mg}+$ Paracetamol $325 \mathrm{mg} 1$ tablet per day for 3 days. If the patient complains about pain stronger than or equal to 4 point of the numeric rating scale (NRS; Figure 1), Ketorolac 1 tablet (maximum 2 times a day) was administered as a rescue dose.

\section{Surgical technique}

The surgical technique used in this study was the Colpimodified Marmar subinguinal varicocelectomy combined with antegrade intraoperative sclerotherapy of venous vessels (9).

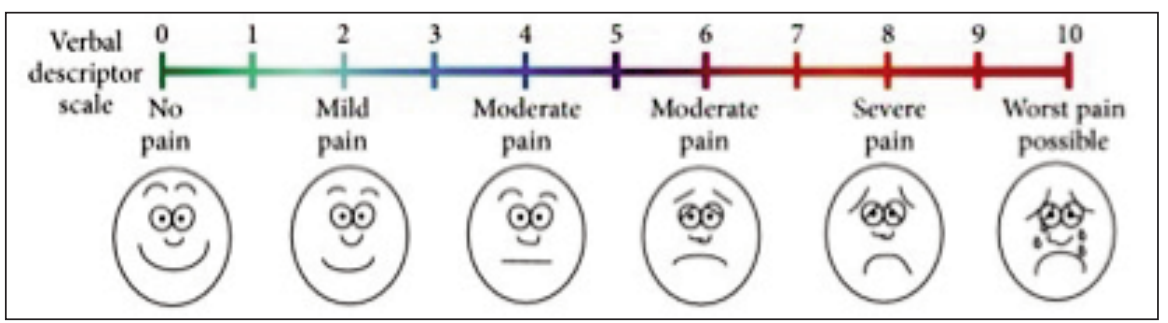

The technique is performed with a $2-3 \mathrm{~cm}$ subinguinal incision at the level of the superficial inguinal ring. After exposition of the spermatic cord, a vein in the spermatic cord outside the peri-arterial venous plexus is identified and isolated. The proximal and distal parts of the spermatic cord are clamped and the identified vein is cannulated using a $25 \mathrm{G}$ butterfly needle. Afterwards, $1.5-3 \mathrm{ml}$ of $3 \%$ atoxysclerol plus $0.5 \mathrm{ml}$ of air is injected; a migration of the sclerosing agent into all visible veins of the spermatic plexus is observed as the movement of the air bubbles. The cannulated vein is then ligated to avoid sclerosing agent leakage. The clamping is released about 8 minutes after the injection. The spermatic and superficial layers are then sutured. Skin is closed in a resorbable subcuticular fashion. An ice pack on the wound is always left in situ.

\section{Follow-up}

All participants were evaluated before surgery, in the first 3 post-operative hours, at 4 days, 1 month and 3 months after the intervention.

The following clinical parameters were considered: time elapsed between the induction of anesthesia and the first urination; time elapsed between the induction of anesthesia and the first walking; pain NRS, pain NRS on movement (NRSm), and any occurred complications. Patients filled a self-assessment diary to update about pain at rest and in movement twice a day for the first 3 post-operative days and once on the $4^{\text {th }}$ post-operative day.

If a patient still complained of NRS pain $\geq 4$, a further outpatient re-evaluation was carried out.

\section{Statistical analysis}

The main outcomes of this study was to compare the two groups in terms of the time from surgery to discharge and reported post-operative pain according to the NRS. Differences between the two groups were tested with the Student t-Test for data with normal distribution, while the Mann-Whitney test was applied for non-normal distributed data. All measured parameters are reported as mean \pm standard deviation (SD). All analyses were performed with the Med-Calc software - version 12.2.1.

\section{RESULTS}

Overall, patients had a mean age of 25 years \pm 5 and a mean body mass index (BMI) of $21 \mathrm{~kg} / \mathrm{m}^{2} \pm 2$. The mean length of surgery was $38 \mathrm{~min} \pm 12$ (Table 1 ).

The mean time elapsed between the induction of anesthesia and the first urination was $240 \mathrm{~min}$ in group A and $210 \mathrm{~min}$ in group B $(\mathrm{p}=0.02)$; the time elapsed until the first walking was $230 \mathrm{~min}$ in group $\mathrm{A}$ and 220 min in group $B(p=0.6)$. Only 5 patients were able to stand up and walk before 120 minutes in Group B vs. no one in Group A.

Figure 1.

The Numeric Rating Scale for post-operative pain reporting. 
Table 1.

Baseline characteristics of both groups.

\begin{tabular}{|l|c|c|c|}
\hline & Group a $(\mathbf{n}=\mathbf{3 2})$ & Group $\mathbf{b}(\mathbf{n}=\mathbf{3 1})$ & $\mathbf{p}$-value \\
\hline Age (years) & $25 \pm 4$ & $25 \pm 6$ & 1 \\
\hline BMI $\left(\mathrm{kg} / \mathbf{m}^{2}\right)$ & $22 \pm 2$ & $21 \pm 2$ & 0.051 \\
\hline Duration (min) & $38 \pm 14$ & $39 \pm 11$ & 0.7 \\
\hline ASA I & 25 & 29 & 0.2 \\
\hline \multicolumn{2}{|l}{ ASA = American Society of Anesthesiologists; BMI = body mass index. } \\
\hline
\end{tabular}

Table 2.

Reported pain score over the first 4 post-operative days.

\begin{tabular}{|l|c|c|c|c|c|}
\hline & $\begin{array}{c}\text { NRS } \\
\text { Group A }\end{array}$ & $\begin{array}{c}\text { NRS } \\
\text { Group B }\end{array}$ & $\begin{array}{c}\text { NRSm } \\
\text { Group A }\end{array}$ & $\begin{array}{c}\text { NRSm } \\
\text { Group B }\end{array}$ & p-value \\
\hline 3 hrs post-surgery & 1 & 1 & 1 & 0 & 0.1 \\
\hline I day 8 am & 7 & 3 & 12 & 8 & $<0.05$ \\
\hline I day 8 pm & 6 & 3 & 9 & 7 & $<0.05$ \\
\hline II day 8 am & 4 & 2 & 9 & 5 & $<0.05$ \\
\hline II day 8 pm & 4 & 2 & 7 & 3 & $<0.05$ \\
\hline III day 8 am & 2 & 0 & 4 & 2 & $<0.05$ \\
\hline III day 8 pm & 2 & 0 & 4 & 2 & $<0.05$ \\
\hline IV day & 0 & 0 & 2 & 1 & $<0.05$ \\
\hline NRS = Numeric Rating Scale.
\end{tabular}

The time elapsed between the induction of anesthesia and the patient discharge was $260 \mathrm{~min}$ in group A and 250 min in group B $(\mathrm{p}=0.1)$.

We then assessed the severity of pain in the first 3 postoperative hours at rest and after mobilization with the NRS: both groups reported a score of 1 at rest, while an NRS score of 1 at movement was reported only for the group A (NRSm) (Table 2).

The NRS scores during the first 4 days after surgery are reported in Table 2. Mean score for Group B were significantly lower at all time-points. None of the patients required rescue therapy and none experienced complications related to the prescribed therapy.

No early or late surgical complications or treatment failures were reported.

\section{Discussion}

Varicocele is highly prevalent in the young male population and frequently associated with male infertility due to semen impairment. (13) For these reason, surgical correction is often required to improve the chance of conception.

As there are various approaches for surgical correction, it is evident from current literature that each technique has its strengths and limitations.

The Marmar technique modified by Colpi is a hybrid between two different surgical approaches: it provides a subinguinal access to the spermatic cord, as in the varicocelectomy according to Marmar; on the other hand, it implies the embolization of the spermatic plexus vessels through the antegrade injection of a sclerosing agent, as per Tauber technique. The original depiction of the technique is performed under general anesthesia and there are no specific trials evaluating the feasibility of a local anesthesia approach $(9,10,14)$.

This local ansesthesia approach could have numerous advantages in an outpatient surgery setting: it does not require tracheal intubation, it allows the reduction of postoperative pain and leads to a reduction in costs, given the smaller number of drugs and assistance required. Finally, postoperative drowsiness is rare after local as compared to general anesthesia, and therefore the patient is usually more oriented and relaxed (15).

When comparing the times from surgery to discharge, we did not find any significative difference between the two groups. Therefore, our data suggest the non-inferiority of a local approach compared to a general anesthesia approach for this surgery.

Regarding the pain evaluation in the first 3 post-operative hours, we noticed significantly lower NRS score for patients receiving local anesthesia. This result is correlated to the average duration of Chirocaine for nerve blocks (analgesic coverage up to 17 hours), thanks to which patients enjoy excellent analgesia with no need of additional drugs. Similarly, lower pain was reported during the first 4 postoperative days with local anesthesia as compared to general.

Our results are in line with the literature. Nordin et al., in 2003, evaluated acceptance, satisfaction, and quality of life in patients performing hernia repair, respectively under genenal, regional and local anestehsia. The authors found that, patients in the local anesthesia group first felt pain significantly later than patients in the other two groups and they also required less analgesics during the first postoperative day (16). In 2012, Kadihasanoglu et al. presented a prospective randomized trial evaluating the feasibility of local anesthesia for varicocelectomy in place of spinal anesthesia. Sixty men with varicocele were included in the study, and the evaluation of pain during and after surgery was determined using the visual analogue scale. Pain scores between the 2 groups did not differ significantly at $2,4,6,8,12$, or 24 hours after surgery. A positive correlation was found between the duration of symptoms and the visual analogue scale score at 24 hours postoperatively. The mean dosage of injected diclofenac was $46.5 \pm 23.3 \mathrm{mg}$ and $32 \pm 28.15 \mathrm{mg}$ in the spinal and local anesthesia groups, respectively. The spinal group developed more postoperative complications, such as urinary retention, postspinal backache, headache, hypotension, and delayed mobilization. In line with our findings they concluded that local anesthesia is an effective, simple, and safe approach for subinguinal varicocelectomy with lower morbidity and fast recovery (17). Likewise, Manaf et al. compared local and general anesthesia in patients who had undergone different andrological procedures including varicocelectomy.

They concluded that office-based andrological procedures using local approach could be successfully performed without compromising surgical technique and post-operative outcomes while significantly reducing the overall costs for both the patient and the healthcare system (18).

The main limitation of this study is the relatively small number of included patients, although we were able to detect a statistically significant difference between groups. Moreover, patients were non-randomized to different treatments thus introducing a potential selection bias. Further trials are needed to confirm our findings. 


\section{Conclusions}

Local anesthesia for varicocele repair using a subinguinal approach is feasible and safe, providing a better control of early postoperative pain as compared to general anesthesia. and a faster recovery without experiencing significant complications. Further larger studies are needed to confirm our positive findings.

\section{References}

1. Dubin L, Amelar RD. Etiologic factors in 1294 consecutive cases of male infertility. Fertil Steril. 1971; 22:469-74.

2. Jarow JP. Effects of varicocele on male fertility. Hum Reprod Update. 2001; 7:59-64.

3. Salonia A, Bettocchi C, Carvalho J, et al. EAU Guidelines on Sexual and Reproductive Health 2020.

4. Miyaoka R, Esteves SC. A critical appraisal on the role of varicocele in male infertility. Adv Urol. 2012; 2012:597495.

5. Dabaja AA, Goldstein M. When is a varicocele repair indicated: the dilemma of hypogonadism and erectile dysfunction? Asian J Androl. 2016; 18:213-6.

6. Marmar JL. The evolution and refinements of varicocele surgery. Asian J Androl. 2016; 18:171-8.

7. Ficarra V, Crestani A, Novara G, Mirone V. Varicocele repair for infertility: what is the evidence? Curr Opin Urol. 2012; 22:489-94.

8. Baazeem A, Belzile E, Ciampi A, et al. Varicocele and male factor infertility treatment: a new meta-analysis and review of the role of varicocele repair. Eur Urol. 2011; 60:796-808.
9. Colpi GM, Carmignani L, Nerva F, et al. Surgical treatment of varicocele by a subinguinal approach combined with antegrade intraoperative sclerotherapy of venous vessels. BJU Int. 2006; 97:142-5.

10. Marmar JL, Kim Y. Subinguinal microsurgical varicocelectomy: a technical critique and statistical analysis of semen and pregnancy data. J Urol. 1994; 152:1127-32.

11. Tauber R, Johnsen N. Antegrade scrotal sclerotherapy for the treatment of varicocele: technique and late results. J Urol. 1994; 151:386-90.

12. Chazapis M, Walker EM, Rooms MA, et al. Measuring quality of recovery-15 after day case surgery. Br J Anaesth. 2016; 116:241-8.

13. Jensen CFS, Østergren P, Dupree JM, et al. Varicocele and male infertility. Nat Rev Urol. 2017; 14:523-533

14. Colpi GM, Carmignani L, Bozzini G, Picozzi S. Surgical subinguinal approach to varicocele combined with antegrade intraoperative sclerosis of venous vessels. Surg Innov. 2012; 19:252-7

15. Rivat C, Bollag L, Richebé P. Mechanisms of regional anaesthesia protection against hyperalgesia and pain chronicization. Curr Opin Anaesthesiol. 2013; 26:621-5

16. Nordin P, Hernell H, Unosson M, et al. Type of anaesthesia and patient acceptance in groin hernia repair: a multicentre randomised trial. Hernia. 2004; 8:220-5.

17. Kadihasanoglu M, Karaguzel E, Kacar CK, et al. Local or spinal anesthesia in subinguinal varicocelectomy: a prospective randomized trial. Urology. 2012; 80:9-14

18. Alom M, Ziegelmann M, Savage J, et al. Office-based andrology and male infertility procedures-a cost-effective alternative. Transl Androl Urol. 2017; 6:761-772.

\section{Correspondence}

Giovannni Saredi, MD

giovanni.saredi@asst-settelaghi.it

Fabrizio I. Scroppo, MD

Paolo Capogrosso, MD

Paolo Maggio, MD

Giuseppe Gianesini, MD

Department of Urology and Andrology; Circolo and Fondazione Macchi Hospital,

Varese Italy

Giacomo Maria Pirola, MD

Lorenzo Capone, MD

Department of Urology, USL Toscana Sud Est, San Donato Hospital, Arezzo, Italy

Andrea Pacchetti, MD

Department of Urology, San Martino Hospital, University of Genova, Italy

Giulio Carcano, MD

Federico Dehò, MD

University of Insubria, Varese, Italy 